\title{
Design and Realization of the Train Simulation Test System
}

\author{
Wanting Zhao ${ }^{1+}$, Xiangxian Chen ${ }^{1}$, Zhujun Ling ${ }^{2}$, Zhenpeng $\mathrm{Nie}^{2}$, Wenbing Tang ${ }^{2}$, Guodong \\ Teng $^{2}$ and Lanqing $\mathrm{Hu}^{2}$ \\ ${ }^{1}$ Department of Instrumentation Science and Engineering, Zhejiang University, Hangzhou, China \\ ${ }^{2}$ Research and Development Center, Zhejiang United Science and Technology Co., Ltd, Hangzhou, China
}

\begin{abstract}
CBTC system is the most popular system in rail transit signaling system. However, as it is the rail train as an object, it takes a lot resource to conduct the investigation under a real situation. Therefore, to develop a CBTC system in lab-based environment is essential. The carborne operation control system is a central system of rail transit control system. It is of most important to protect the safety of the rail train. Therefore, the safety and reality of the carborne operation control system directly influent the whole system's operation and the safety and the efficiency of the train. The current study is focusing on the design and the investigation of the carborne operation control system, which is the central system of the rail transit signaling system.
\end{abstract}

Keywords: rail train transit, CBTC, Carborne, control, simulation

\section{Introduction}

With the fast development of the computer technology, communication technology and the controlling technology, a replacement of the current control system with a comprehensive utilization of these three technologies is becoming an increasing hot topic. The core of this comprehensive utilization is the communication technology [1]-[2]. It generates the Communication Based Train Control [3]-[5]. Communication Based Train Control system is a leading technology of the city railway transit technology. Because of the CBTC has a better safety, stability and communication efficiency, it become a new trend of the future development of the rail transit [6]-[8]. CBTC achieves movement blocking and increasing efficiency of the communication through the advanced location technology of the rail train, safety management technology, as well as wireless communication technology. According to the IEEE1474.1, the typical CBTC system composes Carborne Controller, CC system, Zone controller, ZC system, Computer Based Interlocking, CBI, Automatic Train Supervision, ATS, and other systems.

The CBTC system is the most central controller system of the rail transit controller system. It is the most important part to protect the safety of the rail train transit. The safety and reliability of the CBTC directly affect the whole system safety and efficiently. It is located on the rail train, and is responsible for the data collection and transition from the speed sensor, acceleration sensor, Doppler radar, beacon finder. It achieves the control of the rail train safety, protection of speeding, car door open control, automatic communication between stations, speed adjustment and the brake system. It is the basement of the rail train transit safety.

However, as the huge size of the rail train, the cost will be too much if we use the real train and its transit trail to conduct the study. And it is not safety as well. Therefore, to develop a lab-based rail train controller test system is very important and urgent. Under he current situation of the rail way transition investigation, our study focus on the design and development of a simulation system based on the study of CBTC system.

Corresponding author. Tel.: +8617816858876 .

E-mail address: wtzhao@zju.edu.cn. 


\section{System Description}

Because the carborne operation control system is the most central and important system in the rail train control system, its safety and reliability is of great importance to the safety of the entire rail train. The carborne control system includes Carborne Controller and its outside controller. And its outside controller includes speed sensor, acceleration sensor Doppler radar beacon finder, antenna, driver control monitor and other parts. The carborne controller and its outside simulation modules are illustrated as Fig 1:

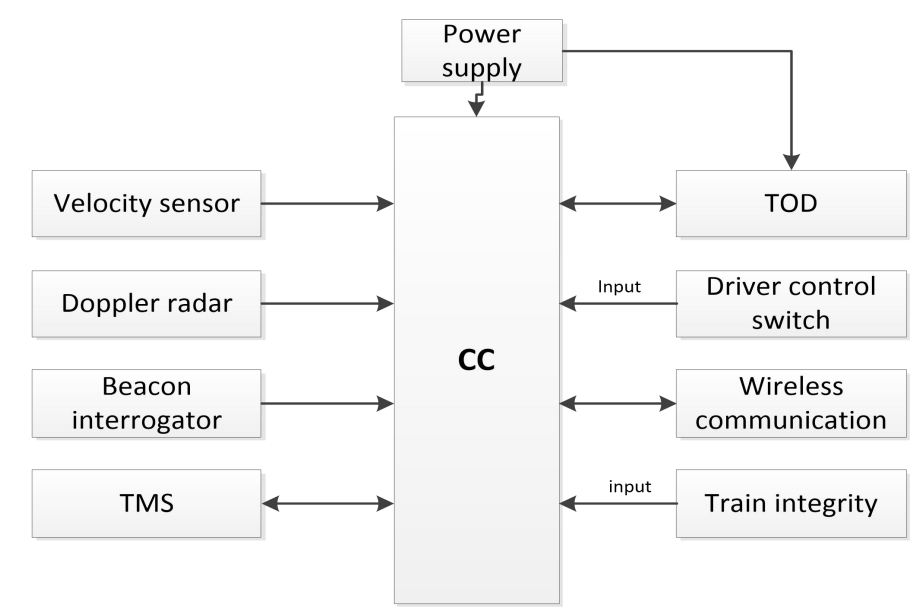

Fig. 1: Carborne controller and its outside interfaces

The carborne controller provides digital output signal interface, digital input signal interface, speed sensor interface, beacon finder interface, and Doppler radar interface and IO interface. To stimulate the carborne controller equipment, people need to achieve the simulation of the software and hardware. The simulation of carborne controller system's physical interfaces includes switch and analog quantity. The analog quantity mainly includes BTM, TMS, radar and speedometer. It is achieved by the simulation of the main control board.

The simulation of the software will be based on the speed value, acceleration value, beacon finder value that calculated through the train running model. The simulation of the hardware will convert the data above to the hardware output: the speed value will be transferred as speed of the data pulse output, Doppler radar will be transferred as real digit and analog voltage output, beacon finder digit will be transferred to serial output.

\section{General Design of Train Simulation System}

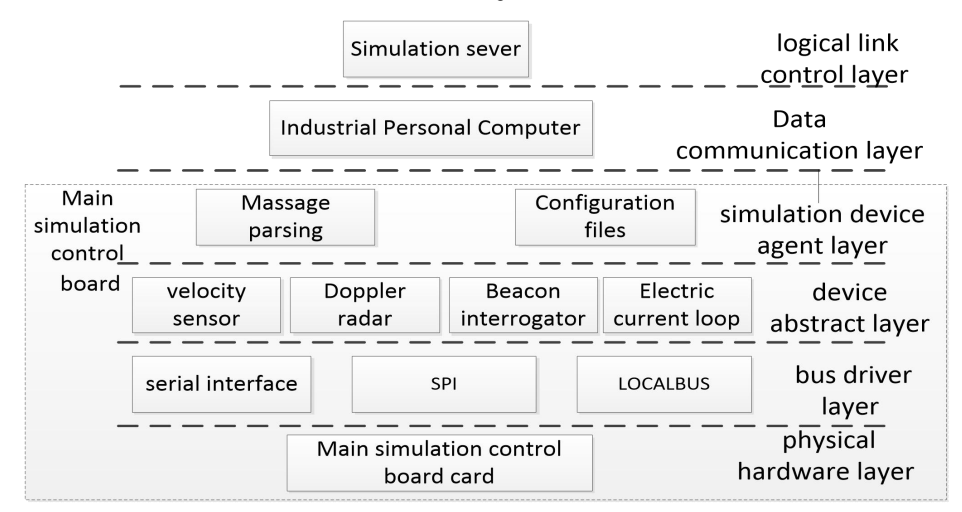

Fig. 2: Overall structure of train simulation system

When the CBTC train control system is used in the train controller CC, it is necessary to transmit the collected peripheral sensor device data such as speed sensor, Doppler radar, beacon and other data to CC to perform the corresponding operation and output. The hardware simulation part of the train simulation system provides the corresponding physical interface for these peripheral sensors to collect and output real-time analog and serial data. As the simulation software part of the sensor analog signal has problems of a 
numerical error and the level signal which does not match, it is necessary to have level matching and precision calibration by the software configuration using the simulation master GPIO. The system simulation software part outputs the corresponding message through the simulation of the peripheral sensor, and drives the main control simulation board to produce the real physical signal.

\subsection{Design of hardware}

As the core processing board of the train simulation system, the main control Simulation board inputs and outputs data through the serial ports of software layer and board's physical layer. The main control simulation board mainly includes the core processing board (Vital Computer Board) and the simulation board, which are stacked on the 6U's simulation floor.VC board mainly achieves the interaction of serial data, FPGA controlling and other functions, while simulation board mainly completes the output of speed meter pulse signal and PWM signal and others. The hardware architecture of train simulation test system is shown in Figure 3:



Fig. 3: The hardware architecture of train simulation test system

\subsection{Design of software}

According to the overall structure of the train simulation system, the entire simulation system will be roughly abstracted into two parts, which consider the bus driver layer as a boundary, the industrial computer simulation software section that has data interaction with the upper software, and the main control simulation board software section that down drives physical interface of the main control simulation board.

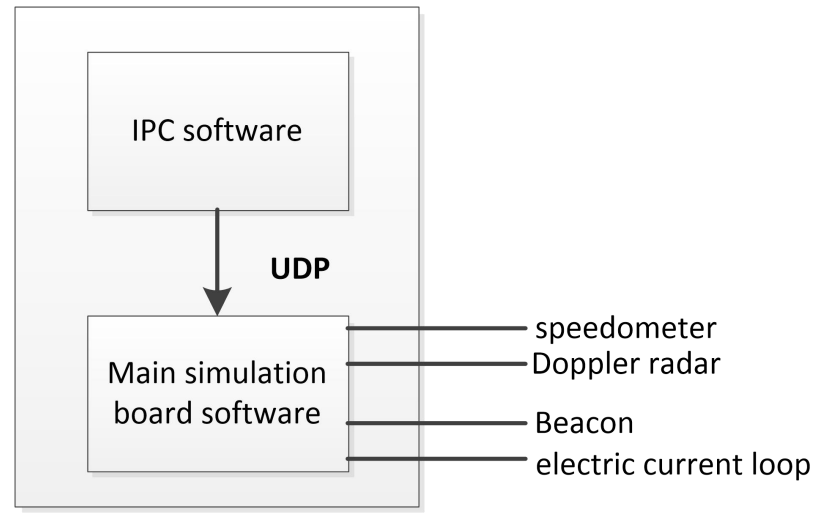

Fig. 4: Software architecture diagram of simulation test system

When the main simulation software runs on the industrial computer, the collection and drive of the IO signal is controlled by the industrial computer simulation software. Here we only discuss the underlying driver software used in the main control simulation board. The software part of the main control simulation board communicates with the industrial computer software through the network UDP protocol. The main control simulation board is used to collect and output real-time analog and serial data. Simulation test system software architecture is shown in Figure 4: 


\section{Test Analysis}

The train simulation system realizes software simulation and hardware simulation respectively. Which is specifically composed of hardware devices, VxWorks operating systems that are responsible for managing hardware devices, software running at the system level, and applications running on system-level software. Among them, the software simulation will achieve the simulation of all the software based on the speed value, acceleration value, beacon query data that calculated by the train simulation model. Hardware simulation will change the above data into hardware output, the speed value into the actual speed pulse output, query data into the serial output, the query host interface. The application program can realize the external communication through the Ethernet port of the train system. And the physical interface of the hardware device is connected with the sensor interface of the simulation system to make up the physical single circuit. The overall test module of the train system is divided as shown in Fig 5.

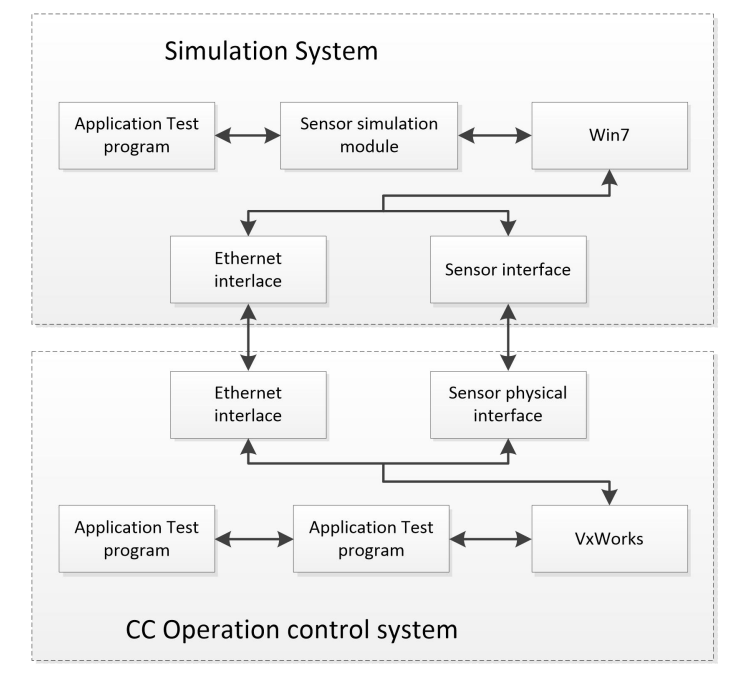

Fig. 5: Division of test module of train simulation system

\section{Summary}

Urban rail train transit system development is of great importance of the urban economic development. Currently, the rail train transit is on its way of being automatic and intelligent. The train operation control system, as the central part of the rail train transit system, is the foundation of the safety, reliability, and stability of the system. As an important way to test the train operation control system, the conduction of the simulation system can solve the problem of site testing of a control system. The current study is about the design and testing of the simulation system. The system, according to the test, doesn't need to change the conduction and connector. The only change needed is the parameter of the connector. The design of this simulation system is not only cost saving but also improve the efficiency of the testing.

\section{Acknowledgements}

This work is supported by the funding from the National Science and Technology Infrastructure Program of China under Grant 2015BAG19B03

\section{References}

[1] Liulin, Chen sodium, Chen Jihua. Study on the Evaluation Index System of Urban Rail Transit Sustainable Development [J]. Journal of Southwest Jiaotong University (Social Science Edition), 2008 (5).

[2] Zeng Xiaoqing, Wang Changlin, Zhang Shijing. Communication-based rail transit operation control [M]. Shanghai: Tongji University Press, 2007. 12-22

[3] Zhang Yannan. Based on the GA train automatic adjustment algorithm in the CBTC system application research [D]. Master's degree thesis, Beijing Jiaotong University, 2008.

[4] Xing Hongxia. Analysis of the closed system of automatic control system for urban rail transit [J]. Journal of Railway Applications. 2008 (07). 
[5] Zhai Yongqiang, Zhang Wei.Analysis of three kinds of occlusion system based on ATC system [J]. Modern Urban Rail Transit. 2011 (03): 87-89.

[6] IEEE Std 1474.1-1990."IEEE Standard for Communication-Based Train Control Performance and Functional Requirements, "Institute of Electrical and Electronics Engineers, Inc., New York[S].1999.

[7] Di Peng, urban rail transit ATC simulation system design [D]. Master's degree thesis, Nanjing University of Science and Technology, 2010.

[8] He Chengcai, mobile occlusion system in the rail transit control advantages [J]. Journal of Zhengzhou Railway Vocational and Technical College, 2012 (2): 6-8 